\title{
$\widehat{A}$ Madridge \\ madridge Journal of Dentistry and Oral Surgery
}

Interconnecting Scientific World

Open Access

\section{Primary Intraosseous Carcinoma Arising on Top of Recurrent Kerato-Cystic Odontogenic Tumor in a Young Male Patient}

\author{
Amjad Mohammed Al Abdul Mohsen ${ }^{1 \star}$, Manal Ibrahim Elnouaem ${ }^{2,3}$, and Omneya Ramadan Mahmoud ${ }^{4}$ \\ ${ }^{I}$ College of Dentistry, Princess Nourah Bint Abdulrahman University, Riyadh, Kingdom of Saudi Arabia \\ ${ }^{2}$ Professor of Oral Pathology, Faculty of Dentistry, Alexandria University, Egypt \\ ${ }^{3}$ Faculty of Dentistry, Princess Nourah Bint AddulRahman University, Riyadh, Kingdom of Saudi Arabia \\ ${ }^{4}$ Lecturer of Oral Pathology, Faculty of Dentistry, Alexandria University, Egypt
}

\section{Article Info}

\section{*Corresponding author: \\ Amjad Mohammed Al Abdul Mohsen College of Dentistry \\ Princess Nourah Bint Abdulrahman University Riyadh \\ Kingdom of Saudi Arabia \\ E-mail: amjad.abdlmohsen@hotmail.com}

Received: November 28, 2016

Accepted: December 21, 2016

Published: March 29, 2017

Citation: Al Abdul Mohsen AM, Elnouaem MI, Mahmoud OR. Primary Intraosseous Carcinoma Arising on Top of Recurrent Kerato-Cystic Odontogenic Tumor in a Young Male Patient. Madridge J Dent Oral Surg. 2017: 2(1): 47-50.

doi: $10.18689 / \mathrm{mjdl}-1000112$

Copyright: ( $\odot 2017$ The Author(s). This work is licensed under a Creative Commons Attribution 4.0 International License, which permits unrestricted use, distribution, and reproduction in any medium, provided the original work is properly cited.

Published by Madridge Publishers

\begin{abstract}
Introduction: Primary intraosseous squamous cell carcinoma (PIOSCC) is carcinoma that occurs within jaw bones exclusively and is considered a rare condition.

The exact percentage of malignant transformation in the wall of odontogenic cysts is yet unknown, It varies between $0.01 \%$ and $0.02 \%$. Although the process of changing into malignancy is not fully understood, long standing chronic inflammation was reported to be an initiating factor for this transformation.

Case Report: We hereby report a case of recurrent odontogenic keratocyst, (OKC) related to the anterior mandibular region. Panoramic radiograph revealed a large radiolucent area which is partially ill defined in some parts. Histological examination of biopsy revealed malignant transformation with dysplastic changes seen in the epithelial lining and areas of frank invasion. Diagnosis of squamous cell carcinoma on top of odontogenic keratocyst was given.

Conclusion: The diagnosis of primary intra osseus carcinoma was given depending on the histological pictures which showed prominent dysplastic changes in the epithelial lining of the cyst together with areas of invasion into the adjacent connective tissue capsule. it was of poor outcome.
\end{abstract}

Keywords: Odontogenic keratocyst; Mandible; Primary Intraosseous Squamous Cell Carcinoma; Malignant Transformation.

\section{Introduction}

PIOSCC is carcinoma that occurs within jaw bones exclusively is considered a rare condition [1]. It is defined as a squamous cell carcinoma (SCC) arising within jaw bones. The epithelium involved in PIOSCC is the one involved in odontogensis, the lining of odontogenic cysts or that arising de novo from odontogenic epithelial rests [2].

According to the recent WHO Classification of odontogenic tumors, OKC was given the term cystic neoplasm known as keratocystic odontogenic tumor (KCOT); which best describes its aggressive clinical behaviour, associated high growth potential and high recurrence rate [3].

The exact percentage of malignant transformation within the wall of odontogenic cysts is yet unknown, It varies between $0.01 \%$ and $0.02 \%$ [4]. Although the process of changing into malignancy is not fully understood, long standing chronic inflammation was reported to be an initiating factor for this transformation [2]. 
The present case described a histopathologically proven case of PIOSCC arising in a recurrent odontogenic keratocyst in a 25 year old male patient.

\section{Case Report}

A 25-year-old male patient was referred to the MaxilloFacial Department at College of Dentistry in August 2014 with an evident swelling located anteriorly in the mandible. The swelling was previously diagnosed as keratocyst as mentioned in the patient's old records; which contained all the data concerning the surgical procedure.

After the surgical procedure was done, the patient suffered from a swelling and although no sign of ulceration was observed at the surgery site, a submental sinus discharging pus was found. It did not improve even after curettage and bone grafting.

External examination showed a bony swelling accompanied by paresthesia in the anterior region and regional lymphadenopathy. Examination of intra oral region showed an edentulous area with mucosal inflammation.

The related medical history was non contributory. The patient was a heavy smoker with a history of smoking at least 25 cigarettes per day for the last 10 years. Dental examination revealed multiple extractions of the lower anterior teeth. The lower premolars on both sides were endodontically treated with apicectomy.

Panoramic radiograph of the mandible revealed a huge irregular lobulated, expansile osteolytic radiolucent lesion of $3 \times 2 \mathrm{~cm}$ extending from lower right premolar crossing to the lower left premolar also showing thinning of the lower cortical plate of bone (Figure 1).

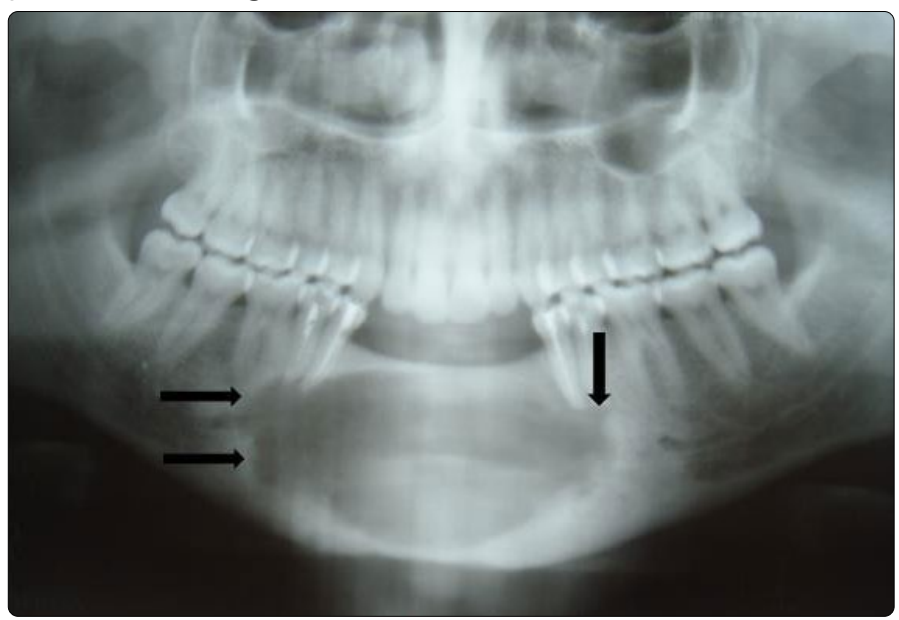

Figure 1: Panoramic radiograph showing a huge osteolytic defect $2 \times 3 \mathrm{~cm}$ in diameter extending from lower right premolar to lower left premolar; the outline is showing an irregular border

Based on the clinical and radiographic examination a provisional diagnosis of recurrent keratocyst or ameloblastoma was given.

Histopathological examination of the biopsy revealed a cystic space lined by corrugated parakeratinized stratified squamous epithelium of 8-10 layers thick (Figure 2) with palisading of basal cells (Figure 3), while other areas of the epithelial lining showed dysplastic features namely hyperchromatism, pleomorphism, basilar hyperplasia, altered $\mathrm{N} / \mathrm{C}$ ratio and loss of polarity of the basal cells (Figure 4).

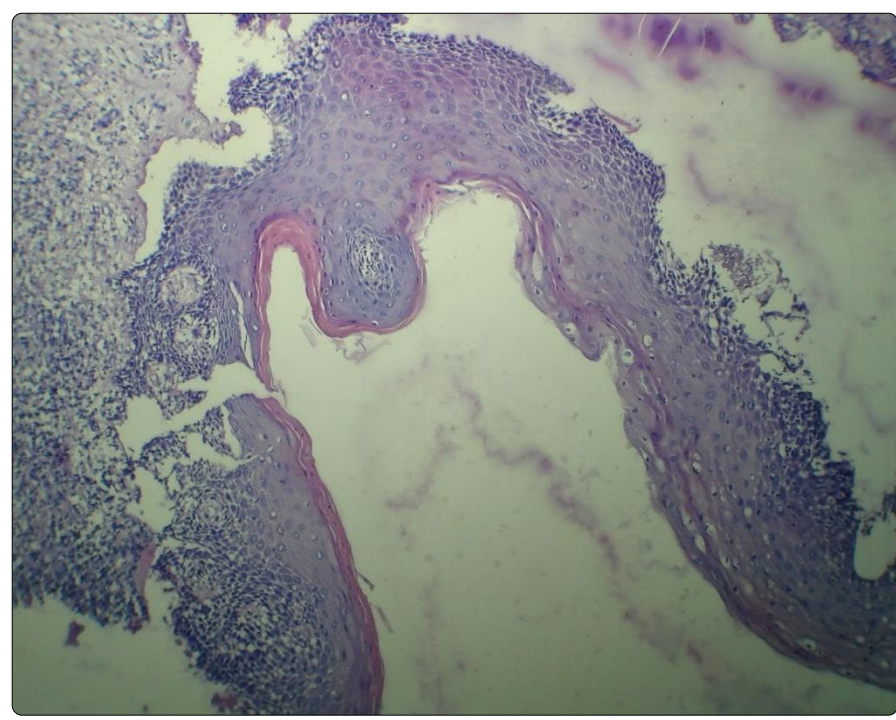

Figure 2: Odontogenic keratocyst showing corrugated parakeratinized stratified squamous epithelium ( $H \& E$ stain $x 200$ )

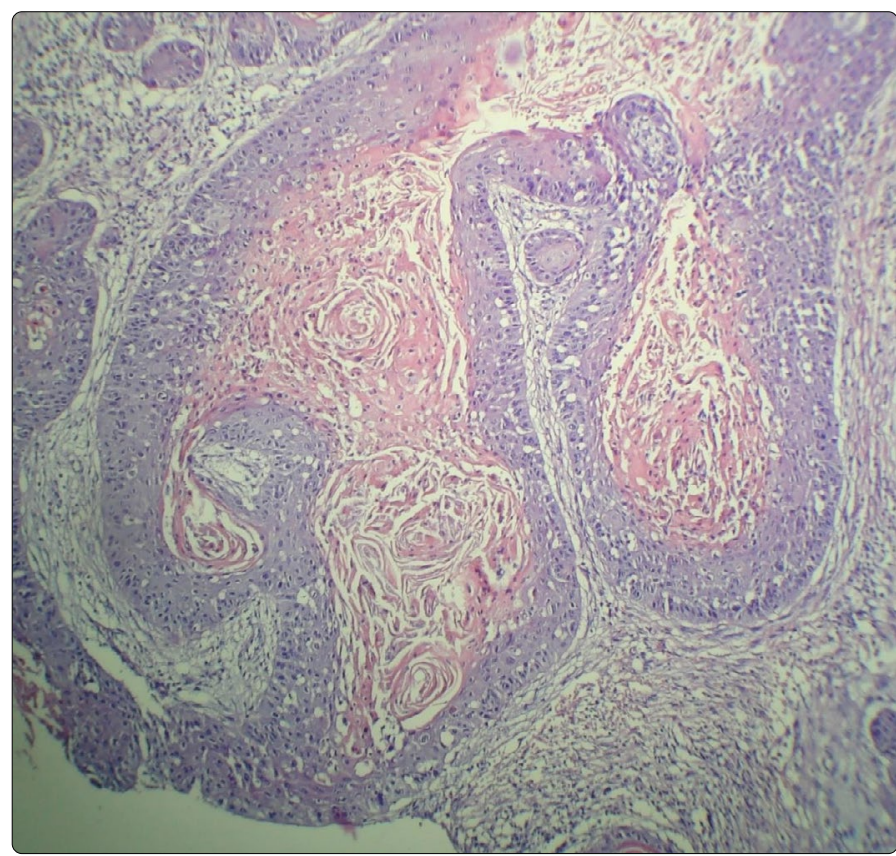

Figure 3: Corrugated epithelial lining showing palisading of the basal cells and cystic lumen filled with keratin (H\&E stain x200). Notice the palisading

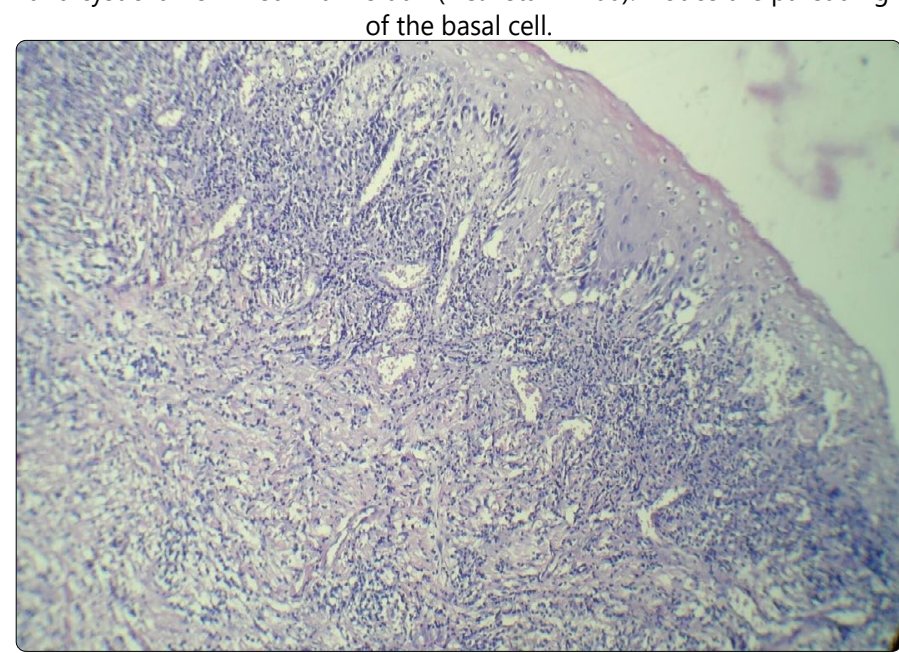

Figure 4: Epithelial lining showing severe dysplastic changes, disruption of basement membrane, point of invasion, severe inflammatory infiltrate and prominent vascularity in the connective tissue beneath (H\&E stain x200) 
The cystic lumen was filled with keratin (Figure 3), disruption of basement membrane and points of invasion were also noted. Severe inflammatory reaction and prominent vascular spaces were seen in close vicinity to the dysplastic epithelium (Figure 4).

The underlying connective tissue demonstrated few keratin pearls, epithelial pearls and nests, surrounded by excessive inflammatory reaction (Figure 5).

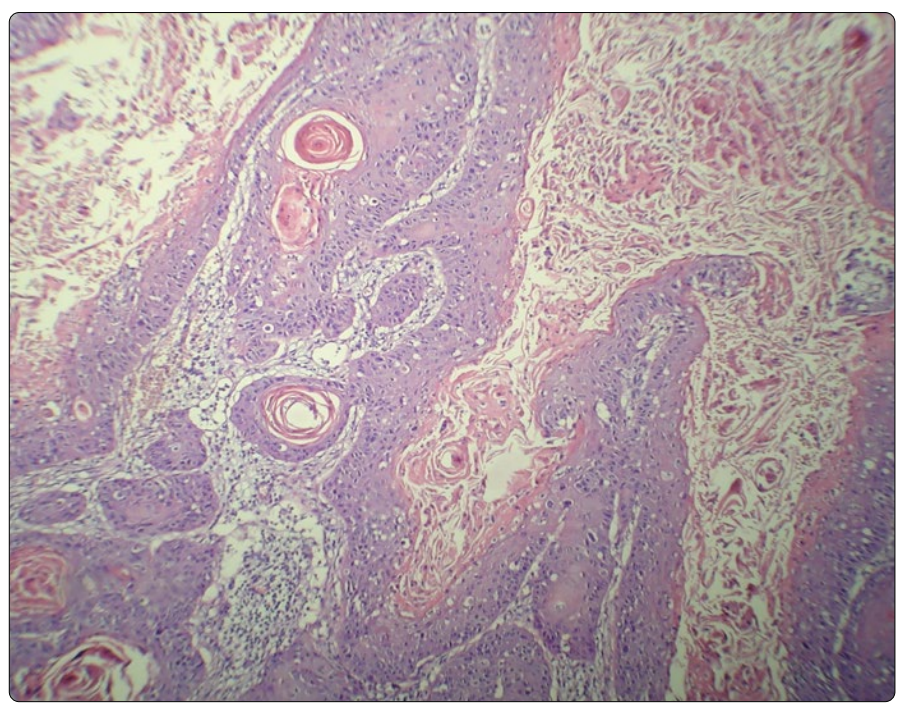

Figure 5: showing the connective tissue having keratin pearls, epithelial pearls and nests together with inflammatory infiltrate. (H\&E stain x200)

The diagnosis was odontogenic keratocyst with malignant transformation into PIOSCC. The patient was admitted for second surgical interference and new reconstruction.

According to surgery report resection was carried from tooth \#36 to tooth \#46 with the overlying skin as well as the mucosa of the anterior $2 / 3$ of the floor of the mouth along with bilateral neck dissection. Reconstruction plates were put using left forearm free flap and wound was closed in layers. The post-surgical follow up revealed impaired vascularity at the flap site, the patient was then readmitted to the operating theatre for exploration and the flap was replaced with Pectoralis major myocutaneous flap. Wound was then closed in layers again.

Six months later, the patient was readmitted for the third time to the Maxillofacial Department with a new recurrence of a swelling at the same site with local metastasis; a new biopsy was taken and again a diagnosis of intraosseous carcinoma was reported.

At this stage the patient received palliative radiotherapy for a month, after which his condition deteriorated and he died because of the complications of metastasis.

\section{Discussion}

PIOSCC was described to be a rare finding within jaw bones [1]. Bodner et al. conducted a retrospective study of 116 cases of PIOSCC between 1938 and 2010 and reported that only 16 cases of these aroused from OKC [2].

Although the exact pathogenesis of PIOSCC is unclear, a series of significant events within the epithelial lining of OKC contributed to the malignant transformation; specifically, keratin metaplasia followed by epithelial hyperplasia and eventually epithelial dysplasia. As reported by Van der Wal et al. those odontogenic cysts with keratin in their lining are more prone and are at a greater risk for malignant changes [5].

In the present case, the biopsy that was taken after the patient's first surgical interference revealed excessive keratin within the cystic cavity, which showed a corrugated epithelial lining featuring dysplastic changes. The underlying connective tissue showed variable inflammatory infiltrate which differed from an area to another. This could have played a factor in malignant transformation confirming the concept that long standing chronic inflammation could be a predisposing factor to malignant transformation [2].

The pooled analysis of the literature of PIOSCC showed that the mean age of the patients is 52.3 years [4]. However, in the present case the patient was in his third decade, thus indicating the possibility of occurrence in younger age groups as was reported by Elnouaem et al. [6] who described a PIOSCC in a 13 year old girl.

Even though the majority of the reported cases showed predilection to mandibular posterior region $[2,4]$, the present case showed anterior mandiblular predilection which is an unusual location of Keratocystic odontogenic tumor.This was in accordance with other studies which described odontogenic keratocystic lesion in the anterior mandibular segment $[7,8]$.

The patient in the present case had symptoms of lymphadenopathy, paresthesia in the anterior mandiblular area in addition to the presence of a draining sinus and a swelling; these symptoms were described previously by Thomas et al. [4].

Jung et al who mentioned similar symptoms attributed the paresthesia to invasion of the neurovascular bundles of the mandible thus mimicking facial neurological pathologies [9].

Radiographicaly, PIOSSC appeared as a radiolucent area with wide differences in appearances. The borders of the intra bony malignant lesions showed regular as well as ill-defined borders depending on the degree and aggressiveness of the malignancy. Cortical plate thinning and erosion was also documented $[4,10]$. In the present case a large radiolucent area was seen which showed smooth borders all over and some irregularity on the lower border, this was not correlated with the histopathological appearance of the present case, where frank invasion was a feature histologically and which was expected to show ill-defined radiolucency. This could be explained as the areas showing the irregular borders were the areas with greatest malignant changes.

The histopathological findings of the present case were similar to many previously reported cases [9], where the epithelial lining of the cyst has undergone malignant changes varying from intraepithelial dysplatic changes to infiltrating squamous cell carcinoma.

The treatment of choice to such tumors is surgery and/or radiation therapy $[1,4]$. Thomas et al. mentioned in his study 
that very poor prognosis of two year survival rate was seen in patients having PIOSCC [4], this was in common with the present case where the patient had a recurrence twice and his condition deteriorated after the second recurrence and he passed away a month later.

No consent form was used as patients coming to the Maxillofacial Surgery Department were considered as educational cases and his or her information was used for research service. The patient in the present case was informed with this information and our main concern here was the histopathological changes occurring in the new recurrent cyst.

\section{Conclusion}

In conclusion, the present case described an unusual presentation of primary intraosseous carcinoma, arising on top of recurrent odontogenic keratocyst in the anterior mandibular region. It was diagnosed as PIOSCC and it was of poor outcome. Proposed treatment was surgery and radiotherapy.

We highly recommend close follow up for patients diagnosed with OKC even after the surgical treatment to find out if any malignant changes have occurred.

\section{Acknowledgements}

We would like to thank Dr. Mervet Moussa; Professor of Oral Pathology, Cairo University for her help and continuous support.

Conflict of Interest: Authors declare no conflict of interest.

\section{References}

1. Saxena C, Aggarwal P, Wadhwan V, Bansal V. Primary intraosseous squamous cell carcinoma in odontogenic keratocyst: A rare entity. J Oral Maxillofac Pathol. 2015; 19(3): 406. doi: 10.4103/0973-029X.174615

2. Bodner L, Manor E, Shear M, vanderWaal I. Primary intraosseous squamous cell carcinoma arising in an odontogenic cyst - a clinicopathologic analysis of 116 reported cases. J Oral Pathol Med. 2011; 40(10): 733-738. doi: 10.1111/j.1600-0714.2011.01058.x

3. Nayak MT, Singh A, Singhvi A, Sharma R. Odontogenic keratocyst: What is in the name?. J Nat Sci Biol Med. 2013; 4(2): 282-5. doi: 10.4103/09769668.116968

4. Thomas G, Pandey M, Mathew A, et al. Primary intraosseous carcinoma of the jaw: Pooled analysis of world literature and report of two new cases. Int J Oral Maxillofac surg. 2001; 30(4): 349-55. doi: http://dx.doi. org/10.1054/ijom.2001.0069

5. Van der Wal KGH, de Visscher JGAM, Eggink HF. Squamous cell carcinoma arising in a residual cyst. Int J Oral Maxillofac surg. 1993; 22(6): 350-352. doi: http://dx.doi.org/10.1016/S0901-5027(05)80665-4

6. Elnouaem $M$, Darwish $Z$, Sharaf $R$. An unusual presentation of odontogenic keratocyst with malignant changes in a 13-year-old girl. International Journal of Case Reports and Images. 2016; 7(10): 657. doi: 10.5348/ijcri-2016116-CR-10704

7. Mohanty S, Gulati U, Mediratta A, Ghosh S. Unilocular radiolucencies of anterior mandible in young patients: A 10 year retrospective study. Natl J Maxillofac Surg. 2013; 4(1): 66-72. doi: 10.4103/0975-5950.117885

8. Veena KM, Rao R, Jagadish Chandra H, Kumar P. Odontogenic Keratocyst looks can be deceptive, causing Endodontic Misdiagnosis. Case Reports in Pathology. 2011; 1-3. doi: http://dx.doi.org/10.1155/2011/159501

9. Jung J, Hwang $H E$, Rae $S$, Hee J. Squamous cell carcinoma arising in an odontogenic cyst. Korean Journal of Oral and Maxillofacial Radiology. 2003; 33(4): 235-238.

10. Nandimath K, Naikmasur VG, Babshet M. Primary Intraosseous carcinoma of Mandible: A case report. Dent Res J. 2011; 8(2): 102-107. 